\title{
Automação como ferramenta para gestão de energia: estudo de caso do Centro de Tecnologia (CT) da Universidade Federal do Rio de Janeiro (UFRJ)'
}

\section{Automation as an energy management tool: case study of Technology Center (CT) of Federal University of Rio de Janeiro (UFRJ)}

\author{
Benevides Pequeno, Luiz Eduardo1; Monteiro, Rafael Sadao2; \\ Pina Filho, Armando Carlos de 3
}

1 Universidade Federal do Rio de Janeiro, Programa de Engenharia Urbana, Escola Politécnica, Av. Athos da Silveira Ramos, 149, Cidade Universitária, 21941-485, Rio de Janeiro - RJ, Brasil, luizeduardobp@poli.ufrj.br

2 Universidade Federal do Rio de Janeiro, Programa de Engenharia Urbana, sadao@poli.ufrj.br

3 Universidade Federal do Rio de Janeiro, Programa de Engenharia Urbana, armando@poli.ufrj.br

\begin{abstract}
RESUMO
A eficiência econômica desempenha um papel particularmente importante no uso institucional dos edifícios. Tornou-se aparente que os custos operacionais são um fator significativo para a Universidade Federal do Rio de Janeiro (UFRJ). As reduções de custos tornaram-se possíveis no mercado de eletricidade graças ao gerenciamento de energia. A automação está facilitando essa integração e comunicação dos componentes na distribuição elétrica, tornando os sistemas elétricos mais confiáveis contra falhas e desperdícios, além de apoiar a eficiência energética graças a funções de medição integradas. Este trabalho apresenta as características do consumo de energia elétrica da UFRJ, além dos detalhes de sua cobrança por parte da concessionária responsável, assim como as ferramentas de automação disponíveis no mercado e como elas podem ser aproveitadas no Centro de Tecnologia (CT). O objetivo principal é identificar de que forma a automação do sistema poderia contribuir na redução dos custos de energia da UFRJ. O método utilizado na pesquisa foi principalmente a visita a campo, além da coleta de dados e pesquisa bibliográfica. Ao final do artigo foram sugeridas as modificações necessárias para a melhoria da gestão de energia no Centro de Tecnologia e apresentadas suas contribuições.
\end{abstract}

Palavras-chave: Automação, Gestão de Energia, Eficiência.

\footnotetext{
${ }^{1}$ BENEVIDES PEQUENO, Luiz Eduardo; MONTEIRO, Rafael Sadao; PINA FILHO, Armando Carlos de. Automação como ferramenta para gestão de energia: estudo de caso do Centro de Tecnologia (CT) da Universidade Federal do Rio de Janeiro (UFRJ). In: II SIMPÓSIO NACIONAL DE GESTÃO E ENGENHARIA URBANA: SINGEURB, 2019, São Paulo. Anais... Porto Alegre: ANTAC, 2019.
} 


\begin{abstract}
Economic efficiency plays a particularly important role in the institutional use of buildings. It has become apparent that operating costs are a significant cost factor for Federal University of Rio de Janeiro (UFRJ). Cost reductions have become possible in the electricity market thanks to power management. Automation is facilitating this integration and communication of components in the electrical distribution, making electrical systems more reliable against faults and waste, and supporting energy efficiency thanks to integrated measurement functions. This work presents UFRJ's electricity consumption characteristics, besides details cost by the responsible utility company, as well as the automation tools available in the market and how they can be used in the Technology Center (CT). The main objective is to identify how the automation could contribute to the reduction of UFRJ's energy costs. The method used in the research was mainly the field visit, besides data collection and bibliographic research. At the end of the article, the necessary changes were suggested for the improvement of the energy management in the Technology Center and presented their contributions.
\end{abstract}

Keywords: Automation, Energy Management, Efficiency.

\title{
1 INTRODUÇÃO
}

A Universidade Federal do Rio de Janeiro (UFRJ) é uma das mais importantes do Brasil e tem como principal Campus a Cidade Universitária na llha do Fundão. Entre os principais centros acadêmicos destaca-se o Centro de Tecnologia (CT), que abriga diversas escolas, institutos e laboratórios. As instalações do CT, em sua maioria, datadas dos anos 70, necessitam de modernização, principalmente no que se refere à rede de energia, devido a sua grande importância para segurança e funcionamento das atividades do local.

Um dos principais problemas do CT são suas elevadas despesas com energia elétrica, sendo um dos centros que mais consome energia na Cidade Universitária. Um dos objetivos da gestão de energia é a redução das despesas com energia elétrica, que representam atualmente $20 \%$ do valor total do orçamento de custeio da UFRJ. Desta forma, iniciativas que promovam o uso racional e eficiente deste recurso se enquadram como prioritárias.

O uso da automação como ferramenta para gestão de energia proporciona o aumento da eficiência energética através do gerenciamento de recursos, reduzindo assim custos em energia elétrica e aumentando a confiabilidade e segurança operacional no fornecimento de energia (DE FARIA e ROCHADEL, 2008; NIŽETIĆ et al., 2019). Assim, o presente estudo tem como objetivo propor o uso da automação como ferramenta para a gestão da energia na UFRJ a fim de controlar melhor o seu consumo e então diminuir o valor gasto nas contas mensais. Alguns recursos incluem: Medidores Inteligentes (Smart Meters), Dispositivos Eletrônicos Inteligentes (IED's) e Redes Elétricas Inteligentes (Smart Grids).

\subsection{Medidores Inteligentes (Smart Meters)}

O Smart Meter (SM) é um medidor de energia avançado que obtém informações do consumo de energia e as fornece à concessionária ou ao operador do sistema para melhorar o monitoramento e faturamento. Com O SM, dados elétricos, tais como tensão, fase e frequência, são medidos e as informações de consumo de energia armazenadas (AMARAL et al., 2014; KUP, 2015).

\subsection{Dispositivos Eletrônicos Inteligentes (IED's)}

Os IED's são microprocessadores com capacidade de trocar dados e sinais de controle com outros dispositivos, usando um link de comunicação. Eles são cruciais nas operações da rede elétrica, pois desempenham as funções de proteção, monitoramento, controle e aquisição 
de dados nas subestações e ao longo dos alimentadores (LEITE, 2015).

\subsection{Redes Elétricas Inteligentes (Smart Grids)}

O Smart Grid baseia-se na utilização intensiva de tecnologia da informação, automação e comunicações para monitoramento e controle da rede elétrica, a qual permitirá a implantação de estratégias de controle e otimização da rede de forma muito mais eficiente que as atualmente em uso (FALCÃO, 2009; DI SANTO et al., 2015).

\section{ENERGIA ELÉTRICA NA UFRJ}

\subsection{Características de Cobrança}

A Light (2016), concessionária de energia elétrica no Rio de Janeiro, divide sua cobrança entre dois grandes grupos tarifários: o Grupo Tarifário "A", formado por clientes que tem fornecimento de energia em tensão igual ou superior a $2,3 \mathrm{kV}$, no caso da UFRJ, e o Grupo Tarifário "B", que inclui unidades consumidoras com fornecimento em tensão inferior a 2,3 kV.

O quilowatt hora ( $\mathrm{kWh}$ ) residencial, do grupo $B$, custa em torno de $R \$ 0,720$, podendo variar dependendo dos tributos incluídos na cobrança, além da questão das bandeiras tarifárias. Para a UFRJ, o custo do kWh varia de $R \$ 0,326$ no horário fora de ponta (HFP) a $R \$ 1,180$ no horário de ponta (HP), entre 17:30h e 20:30h. Portanto, a energia no HP é 3,62 vezes mais cara do que no HFP, evidenciando a importância de se ter meios eficazes para gerenciar o consumo ao longo do dia.

A tarifa para grandes clientes, como é o caso da UFRJ, é composta pelo consumo no HP (em $\mathrm{kWh}$ ), pelo consumo no HFP (em kWh) e pela demanda contratada (em kW). Essa demanda é definida como a potência ativa, e corresponde ao valor que a concessionária deve disponibilizar no ponto de entrega, conforme as condições do contrato de fornecimento, e que deve ser integralmente paga, sendo utilizada ou não (MME, 2011).

\subsection{Demanda Energética na UFRJ}

A despesa da UFRJ com energia elétrica alcançou o valor de $R \$ 25,6$ milhões em 2014. Em 2015, essa despesa teve um aumento de cerca de $80 \%$ mesmo sem crescimento significativo no consumo durante este mesmo ano. Isso ocorreu devido ao "tarifaço", ajustes extraordinários sobre as distribuidoras de energia e um acréscimo para o sistema de bandeiras tarifárias (ANNEL, 2016), porém os gastos continuaram a crescer em 2016 com a expansão do Campus e a inauguração de novas instalações, chegando a alcançar $R \$ 53$ milhões (Figura 1).

Figura 1 - Gasto da UFRJ com energia elétrica entre os anos de 2014-2016.

\begin{tabular}{|l|l|l|l|l|l|l|l|l|l|l|l|l|l|}
\hline Ano & Jan & Fev & Mar & Abr & Mai Jun & Jul & Ago & Set & Out Nov & Dez & Total \\
\hline 2016 & 4,9 & 5,0 & 5,5 & 5,0 & 4,7 & 3,9 & 5,1 & 3,5 & 4,4 & 3,5 & 3,9 & 3,6 & 53,0 \\
\hline 2015 & 2,7 & 3,1 & 4,0 & 4,2 & 3,5 & 3,8 & 3,1 & 3,8 & 4,0 & 4,3 & 4,6 & 4,9 & 46,0 \\
\hline 2014 & 1,8 & 2,5 & 2,5 & 2,2 & 1,9 & 1,8 & 1,6 & 1,8 & 1,9 & 2,2 & 2,6 & 2,8 & 25,6 \\
\hline
\end{tabular}

Fonte: Reitoria da UFRJ.

Uma das principais dificuldades encontradas pela universidade para gerenciamento do consumo, e consequentemente redução do valor das contas de energia elétrica, é sem dúvida controlar sua demanda de forma eficiente. No caso específico do CT, de acordo com a Figura 2, a maior parte do ano, cerca de oito meses, a demanda contratada (linha 
vermelha) é ultrapassada. Essas demandas de ultrapassagens acabam gerando um custo maior na conta de energia devido à multa aplicada pela concessionária de energia.

Figura 2 - Demanda mensal de energia do CT/UFRJ.

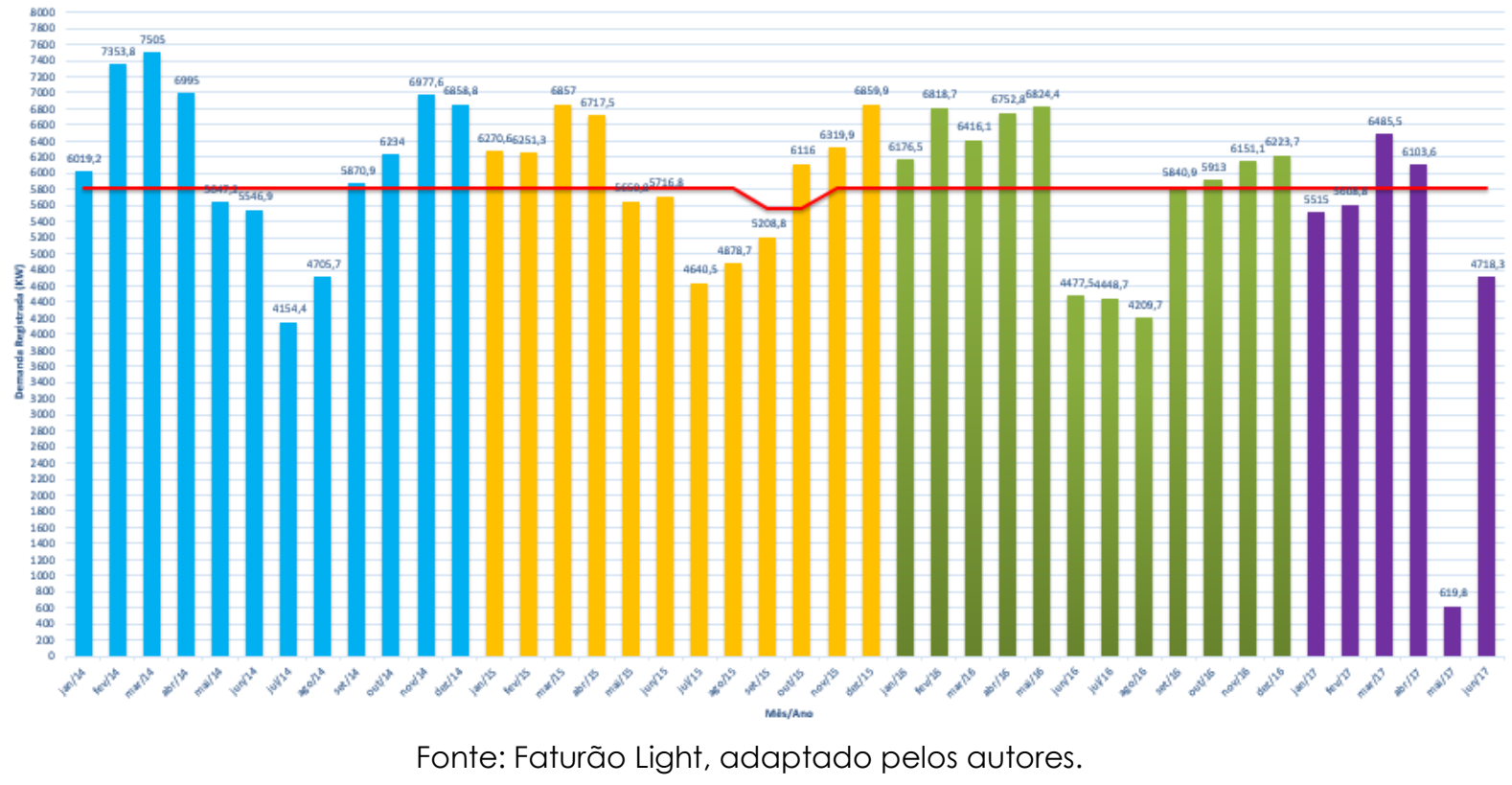

A linha vermelha indica o valor da demanda contratada (KW) para o CT. Conforme observado nas barras coloridas, a demanda energética apresenta um padrão, que se caracteriza por um consumo abaixo do contratado apenas no período do inverno. De fato, no verão, quando o uso dos aparelhos de ar condicionado é mais intenso, a demanda por energia será maior. No entanto, como não existe ainda uma automação eficiente para o gerenciamento do consumo, não se sabe o quanto isso representa no valor total demandado. Essa informação, assim como tantas outras, é fundamental para que se possa definir com maior precisão o valor a ser contratado, e então evitar sua ultrapassagem.

O consumo (kWh) durante o período de medição é o principal componente da conta de energia do CT. O valor gasto para o consumo no HP está na ordem dos $R \$ 220.000,00$, o que somado aos outros consumos totalizou uma conta final de aproximadamente $R \$ 580.000,00$ no mês de junho 2017. Este consumo no HP, que custa mais de três vezes o valor do HFP, possivelmente poderia ser reduzido, uma vez que a grande maioria das instalações do CT funciona de 07:00h às 17:00h, ou seja, dentro do HFP.

\section{APLICAÇÕES PRÁTICAS NO CT/UFRJ}

Em junho de 2017, o CT/UFRJ teve um consumo que representou aproximadamente $18 \%$ da fatura total da Cidade Universitária, conforme mostra a Figura 3. 
Figura 3 - Gasto com energia na UFRJ em junho de 2017.

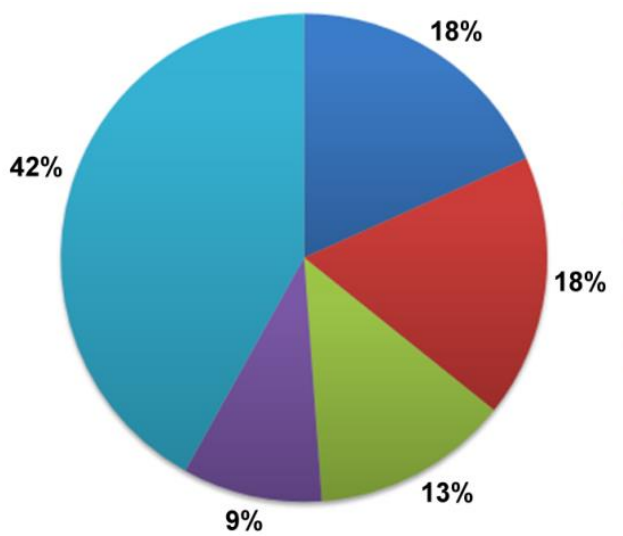

CENTRO TECNOLÓGICO

CCS - BLOCOS A ATÉ L

HOSPITAL UNIVERSITÁRIO

LADETEC

DEMAIS UNIDADES

Fonte: Faturão Light, adaptado pelos autores.

Uma das principais ações para economizar energia é medir detalhadamente o consumo. Entretanto, ter dados de consumo isoladamente não traz eficiência energética nem nenhuma redução de gastos. Medidores de energia instalados isoladamente não possuem retorno caso suas informações não sejam utilizadas corretamente.

A gestão energética eficiente busca minimizar o consumo e maximizar a eficiência energética, aproveitando as informações obtidas de equipamentos multifuncionais de forma planejada e com ações sistêmicas (OLIVEIRA, 2006). Muitas empresas são especializadas em produtos e serviços para aplicações elétricas, controle e automação com destaque para alemã SIEMENS e a francesa SCHNEIDER ELECTRIC.

\section{METODOLOGIA E PROPOSTAS}

Visando a melhoria no gerenciamento do consumo de energia elétrica no CT, sugere-se a instalação de SMs nas unidades de medição (UE1, UE2, UE3), dispositivos eletrônicos inteligentes nas 8 cabines de transferência para os blocos $\mathrm{A}$ a $\mathrm{H}$, e nas 26 subestações, além de dispositivos controladores de energia elétrica nos equipamentos atendidos por estas subestações. A Figura 4 mostra como a rede elétrica do CT estaria distribuída.

Figura 4 - Locais para instalação de equipamentos de medição e controle no CT/UFRJ.

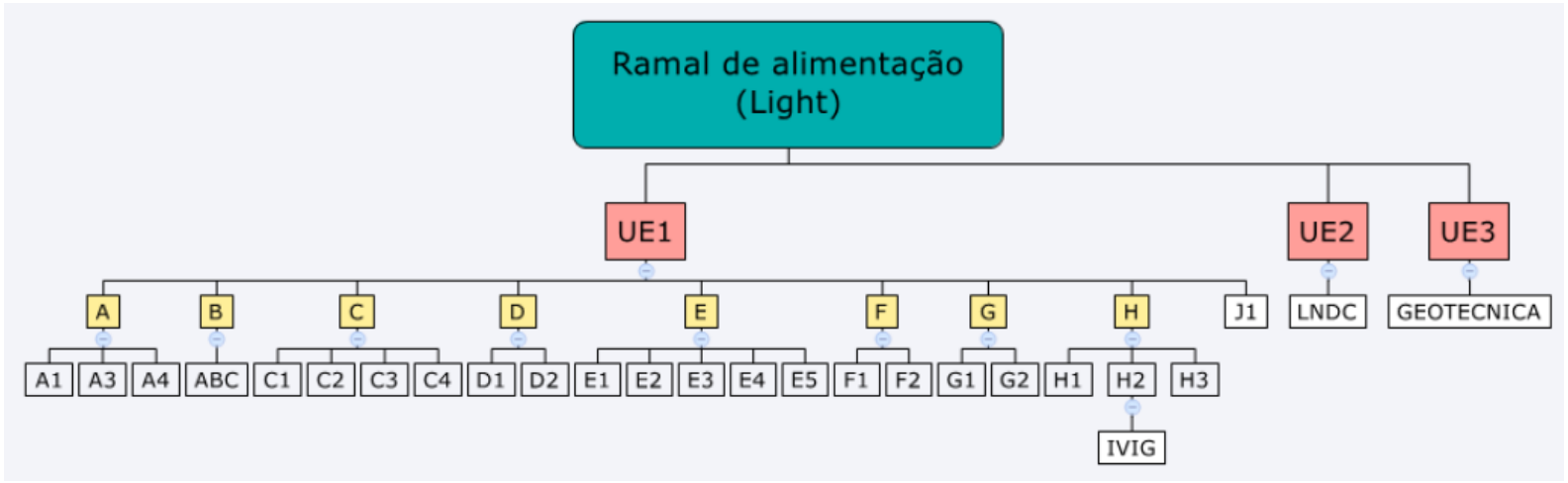

Fonte: EPLAN/CT.

A incorporação dos multimedidores nas UE's é um passo importante para se alcançar a eficiência energética. A instalação desses equipamentos visa conhecer o consumo e mapeálo, buscando os momentos em que ocorrem os picos de consumo e entender em que ponto deve-se aplicar uma gestão de energia. É preciso reavaliar o processo e os dados de medição, verificando se as medidas implementadas são suficientes ou podem ser melhoradas, fechando um ciclo de evolução contínua.

Com a monitoração das cabines de transferência é possível gerenciar a quantidade de 
energia utilizada, controlar a demanda, saber onde estão instaladas as maiores cargas e quando há maior utilização de energia elétrica, além do valor da conta e a qualidade da energia usada.

No caso das subestações, a automação presente no controle de demanda não serve apenas para que se evitem as multas por ultrapassagem, mas que proporcione um controle justo sobre as cargas, possibilitando a diminuição da demanda contratada através de operação programada para descarte de cargas não essenciais atendidas por disjuntores motorizados, que permitem operações remotamente.

Existem equipamentos de automação denominados controladores de demanda capazes de fazer esse gerenciamento de forma automática, que trabalham em sintonia com o medidor da concessionária e identificam continuamente o ritmo de ultrapassagem em cada bloco de medição. Quando necessário, esses equipamentos podem desligar e religar várias cargas para manter a demanda máxima dentro da tolerância de contratação, evitando assim, a incidência de multas.

\section{CONSIDERAÇÕES FINAIS}

Dado o alto valor das contas de energia da UFRJ, fica evidente a necessidade de se obter meios de melhorar sua gestão energética. Os potenciais de redução da fatura mensal do CT passam pelo ajuste da demanda contratada e pelo consumo diário de energia. O uso de técnicas e recursos de automação no processo de gerenciamento do consumo de energia elétrica no prédio do CT na UFRJ poderia contribuir de forma significativa para a redução de seus custos.

Caso fossem adotadas essas soluções para monitoração de energia, seria possível a UFRJ gerenciar a quantidade de energia utilizada, controlar a demanda e o valor da conta. Além disso, os dados de desempenho energético poderiam ser transmitidos através da Web ou rede local. As informações estariam facilmente acessíveis, apoiando estratégias de gestão energética, assim como resolução de problemas e manutenção remota.

Com o acompanhamento dos dados obtidos pelos medidores inteligentes seria possível, por exemplo, analisar detalhadamente os horários e pontos de maior consumo, identificando equipamentos de baixa eficiência energética que poderiam ser substituídos, além de facilitar o escalonamento das cargas, evitando assim as multas por ultrapassagem da demanda contratada.

Concluindo, existe total viabilidade técnica para instalação dos multimedidores a jusante dos transformadores abaixadores, pois os mesmos utilizam apenas sinais de tensão e correntes das fases. Além dos medidores, é necessária a utilização de controladores lógicos programáveis, que comandam as operações de abertura e fechamento de disjuntores de baixa tensão. Todos esses equipamentos devem ser interligados através de uma rede estruturada, sendo que a estimativa do custo de instalação e economia esperada ainda serão avaliados em trabalhos futuros.

\section{REFERÊNCIAS}

AMARAL, H. L. M.; SOUZA, A.N.; GASTALDELLO, D. S.; FERNANDES, F.; VALE, Z. Smart meters as a tool for energy efficiency. In: 11 th IEEE/IAS International Conference on Industry Applications, Juiz de Fora - MG, Brasil, 07-10 dez. 2014.

ANNEL. Agência Nacional de Energia Elétrica. Por Dentro da Conta de Luz, $7^{a}$ Edição. Brasília DF, 2016.

DE FARIA, A. B. M.; ROCHADEL, A. D. Gestão de energia elétrica no campus Darcy Ribeiro:

Rateio e ferramentas. Trabalho de Conclusão de Graduação. Universidade de Brasília, Faculdade de Tecnologia, Departamento de Engenharia Elétrica, Brasília - DF, jul. 2008. 
DI SANTO, K. G.; KANASHIRO, E.; DI SANTO, S. G.; SAIDEL, M. A. A review on smart grids and experiences in Brazil. Renewable and Sustainable Energy Reviews, Vol. 52, 2015, p. 1072-1082.

FALCÃO, D. M. Smart Grid e Microrredes: O Futuro já é Presente. In: VIII Simpósio de Automação de Sistemas Elétricos (SIMPASE). Rio de Janeiro - RJ, 09-14 ago. 2009.

KUP, M. T. Estudo da Medição Inteligente para Consumidores Residenciais no Brasil. Projeto de Graduação. Curso de Engenharia Elétrica da Escola Politécnica da Universidade Federal do Rio de Janeiro, UFRJ. Rio de Janeiro - RJ, 2015.

LEITE, J. B. Desenvolvimento de um Sistema de Gerenciamento de Energia (EMS - Energy Management System) para a Rede Elétrica Inteligente (Smart Grid). Tese de Doutorado. Faculdade de Engenharia da Universidade Estadual Paulista, UNESP. Ilha Solteira, São Paulo, 2015.

LIGHT. Regulamentação para Fornecimento de Energia Elétrica a Consumidores em Média Tensão (RECON), março 2016.

MME. Ministério de Minas e Energia. Manual da Tarifação da Energia Elétrica, agosto 2011.

NIŽETIĆ S.; D JILALI N.; PAPADOPOULOS A.; RODRIGUES J. J. P. C. Smart technologies for promotion of energy efficiency, utilization of sustainable resources and waste management. Journal of Cleaner Production, Vol. 231, 2019, p. 565-591.

OLIVEIRA, L. S. de. Gestão do consumo de energia elétrica no campus da UnB. Dissertação de Mestrado. Universidade de Brasília, Faculdade de Tecnologia, Departamento de Engenharia Elétrica, Brasília - DF, ago. 2006.

SCHNEIDER ELECTRIC. Disponível em: <https://www.se.com/br/pł>. Acesso em: março 2018. SIEMENS. Disponível em: <https://new.siemens.com/br/pt.html>. Acesso em: março 2018. 